\title{
Knockdown of Adenosine Deaminase 1 Acting on dsRNA Inhibits Expression of Proopiomelanocortin in HaCaT Cells
}

\author{
Te-Sheng Chang, Yen-Hsing Lu, and Hsiou-Yu Ding
}

\begin{abstract}
In 2003, ADAR1 (Adenosine Deaminase 1 Acting on dsRNA) was identified as the disease gene for an autosomal dominant disease - dyschromatosis symmetrica hereditaria (DSH) - which is characterized by a mixture of hyperpigmented and hypopigmented macules that are distributed on the dorsal aspects of the extremities and freckle-like macules on the face. To date, more than 100 ADAR1 mutations have been discovered in DSH patients. However, the exact molecular pathogenesis of DSH has not been clearly identified. To investigate the effects of ADAR1 mutations on the signal transduction pathway of melanogenesis, we used the RNA interference (RNAi) method to knockdown ADAR1 in human keratinocyte HaCaT cells, and determined a hormone precursor peptide proopiomelanocortin (POMC) expression in the ADAR1-knockdown HaCaT cells. Based on the results, we found that ADAR1 knockdown inhibits POMC expression in HaCaT cells. The findings of the present study imply that ADAR1 plays an important role in regulating POMC expression in keratinocytes, and possibly, the UV-induced pigmentation of skin.
\end{abstract}

Index Terms-ADAR, DSH, HaCaT, Melanogenesis, Pigmentation, POMC.

\section{INTRODUCTION}

After transcription, the newly formed transcript RNA undergoes a number of modifications, such as 5' capping, splicing, 3' processing, and RNA editing [1]. These processes are mechanisms for generating a diverse set of RNA and protein products from a limited number of DNA genes. The phenomenon of RNA editing was first discovered more than 20 years ago in kine-toplastid protozoa [2]. In the animal kingdom, the most prevalent type of RNA editing that alters one nucleotide to another is mediated by ADAR enzymes; ADARs convert adenosines to inosines $(\mathrm{A} \rightarrow \mathrm{I}$ editing $)$ in double-stranded RNA (dsRNA) substrates. Inosine is recognized as guanosine $(\mathrm{G})$ by the translation and splicing machineries, and thus, ADARs can alter the protein-coding information of mRNA. Noncoding regions of mRNA, such as introns and untranslated regions (UTRs), are also targeted by

Manuscript received April 9, 2012; revised May 30, 2012. This work was supported in part by the National Scientific Council of Taiwan under Grant 100-2221-E-024-003.

T. S. Chang is with the Department of Biological Science and Technology, National University of Tainan, $33 \mathrm{sec} .2$ Shu-Lin St., Tainan, 702, Taiwan (Tel.: +886-6-2606153; fax: +886-6-2606153; e-mail: mozyme2001@gmail.com).

Y. H. Lu is with the Department of Biological Science and Technology, National University of Tainan (e-mail: ab90377@hotmail.com).

H. Y. Ding is with the Institute of Cosmetics Science, Chia Nan University of Pharmacy and Science, $60 \mathrm{sec}$. 1 Erh-Jen RD, Jen-Te, Tainan, 717, Taiwan (Tel: +886-6-2664911; fax: +886-6-2670324; e-mail: hsiou221@yahoo.com.tw).
ADARs, usually within dsRNA formed by the pairing of inverted repetitive elements [3]. ADARs are expressed in a variety of cells and tissues, with the highest expression in the brain and spleen. ADARs are conserved in their adenosine deaminase domain, but differ in their RNA binding domains. ADARs contain two or three dsRNA binding domains (dsRBD), in addition to an adenosine deaminase domain. Human ADAR1 spans $30 \mathrm{~kb}$ and contains 15 exons, which are composed of 1,226 amino acid residues.

Dyschromatosis symmetrica hereditaria (DSH) (OMIM 127400) is an autosomal dominant disease that is characterized by a mixture of hyperpigmented and hypopigmented macules of various sizes on the backs of the hands and feet. The onset of this disease is usually during infancy or childhood, and $73 \%$ of patients develop skin lesions before the age of 6 years [4]. This disease was first described by the Japanese dermatologist Toyama in 1910 . DSH been reported mainly in Japanese and Chinese subjects. Several cases of DSH have also been reported among Korean, Indian, European, and South American individuals. No racial difference in the condition has been observed, but the disorder might be distributed mainly in East Asia. Zhang et al. mapped the DSH locus to chromosome 1q11-1q21 [5]; then, Miyamura et al. determined the locus to be on 1q21.3., where the pathogenic mutations were identified as the gene ADAR1 [6]. To date, more than 100 ADAR1 mutations have been discovered in DSH patients [7].

The fact that DSH is inherited in a dominant fashion indicates a gain-of-function disease mechanism or a dominant negative effect of the mutant proteins on normal ADAR1 function. In mice heterozygous for an ADAR1 null mutation, no phenotype is discernible, whereas homozygous ADAR1 knock-out animals die during early embryogenesis [8], [9]. No pigmentation abnormality is detected in the limbs of $A D A R 1+/-$ mice [10]. Recently, the ADAR1 gene has been shown to play key, direct roles in the regulatory functions of epidermal skin cells [10]. Hence, it will be interesting to see if an ADAR1-specific RNA editing target(s) will emerge in the future that can explain the observed DSH phenotype and disease mechanism.

Until now, the study of DSH has focused on the mutation screening of the DSH families [7]. However, the exact molecular pathogenesis of DSH has not yet been clarified. It is unclear why pigmentation changes in DSH are localized specifically on the exposed parts. Melanin is the pigment responsible for the color of human skin, and tyrosinase (TYR) is known to be a key enzyme that catalyzes the synthesis of melanin in melanocytes [11]. A previous study has reported that some cytokines and growth factors play important regulatory roles in melanogenesis, where the $\alpha$-melanocyte 
stimulating hormone $(\alpha-\mathrm{MSH})$ is the most well-studied hormone [12]. This hormone is derived from the POMC peptide and secreted by skin keratinocytes, where UV is a major factor in inducing this cascade pathway. The mutations of the ADAR1 gene may affect the signal transduction pathway of melanogenesis and change the menalocyte activity, resulting in hyperpigment or hypopigment production in menalocytes. Therefore, it is interesting to see whether the ADAR1 gene product will affect the POMC- $\alpha$ MSH signal pathway. In the present study, we used the RNAi method to knockdown ADAR1 in human keratinocyte HaCaT cells, and determined POMC expression in the ADAR1-knockdown $\mathrm{HaCaT}$ cells. From the results, we demonstrate that ADAR1 knockdown will inhibit POMC expression in $\mathrm{HaCaT}$ cells.

\section{MATERIALS AND METHODS}

\section{A. Cell Cultures}

Human keratinocyte HaCaT cells are a kind gift from Dr. Te-Chang Lee, the Division Coordinator at the Institute of Biomedical Sciences of Academia (Sinica, Taiwan). The cells were cultured in Dulbecco's modified Eagle's medium (DMEM) and supplemented with $10 \%(\mathrm{v} / \mathrm{v})$ fetal bovine serum at $37^{\circ} \mathrm{C}$ in a humidified, $\mathrm{CO}_{2}$-controlled $(5 \%)$ incubator. The cells were seeded at an appropriate cell density in a 24-well or a 6-well plate.

\section{B. ADARl Knockdown in HaCaT Cells}

The shRNA targeting of human ADAR1 was designed according to the methods of Single Oligonucleotide RNAi Technology for Gene Silencing ( the pRNAi vector system for stable shRNA expression), according to the manufacturer's protocol (BioSettia, www.biosettia.com). The ADAR1-specific RNA interference target region was selected according to the ADAR1 genome sequence (GenBank No. NM_001111), and the specific sequences are listed in Table I. All of the siRNA duplexes were chemically synthesized and cloned into the pRNAi vector provided by the kit. The negative control plasmid (RNAi-c) given in the kit did not target any known mammalian gene. The resulting recombinant plasmids were linearized via restriction enzyme PacI before the transfection experiments were performed. Transfection was performed in 6-cm tissue culture dishes using PolyJet ${ }^{\mathrm{TM}}$ In Vitro DNA Transfection Reagent (SignaGen, www.signagen.com), according to the manufacturer's protocol. The transfected cells were washed with warm DMEM containing 10\% FBS $6 \mathrm{~h}$ after transfection, and were cultured in the same medium containing a selection antibiotic.

\section{C. $R T-q P C R$}

Real time reverse-transcription polymerase chain reaction (RT-qPCR) was performed using the ABI 7500 Real Time PCR system (Applied Biosystems, Foster, CA) employed the Fast SYBR ${ }^{\circledR}$ Green Master Mix (Applied Biosystems). Total RNA was extracted using an RNeasy ${ }^{\circledR}$ Mini Kit (Qiagen, Valencia, CA), according to the manufacturer's instructions. The quality of the total RNA sample was evaluated by determining the $\mathrm{OD}_{260} / \mathrm{OD}_{280}$ ratio. To prepare a cDNA pool from each RNA sample, total RNA (2 $\mu \mathrm{g})$ was reverse-transcribed at $42^{\circ} \mathrm{C}$ for $90 \mathrm{~min}$ in the presence of oligo(dT) primers (MD Bio., Taipei) and reverse transcriptase (Roche Molecular Biochemicals, Mannheim). The oligonucleotide primers used for human ADAR1, POMC, and $\beta$-actin are listed in Table II. After the initial incubation of $2 \mathrm{~min}$ at $50^{\circ} \mathrm{C}$, the cDNA was denatured at $95^{\circ} \mathrm{C}$ for $10 \mathrm{~min}$, followed by 40 cycles of PCR $\left(95^{\circ} \mathrm{C}, 15 \mathrm{~s} ; 60^{\circ} \mathrm{C}, 60 \mathrm{~s}\right)$. The mRNA levels of ADAR1 and POMC were normalized using $\beta$-actin as an internal control. The relative mRNA content was calculated by dividing the normalized data by the data from the control reaction.

TABLE I: ADAR1 GENE SEQUENCES FOR RNAI GENE- KNOCKDOWN

\begin{tabular}{|c|c|c|}
\hline Name & $\begin{array}{l}\text { Sequence ( } 5 \text { '-AAAA-sense } \\
\text { seq.-TTGGATCCAA-anti-sense seq.-3') }\end{array}$ & $\begin{array}{l}\text { Locatio } \\
\mathrm{n}\end{array}$ \\
\hline RNAi1 & $\begin{array}{l}\text { 5'-AAAAGTGAGTTAATGAAATACATTGG } \\
\text { ATCCAATGTATTTCATTAACTCAC -3, }\end{array}$ & $\begin{array}{l}2974- \\
2992\end{array}$ \\
\hline RNAi2 & $\begin{array}{l}\text { 5'-AAAAGAACAAAGGATCTTAAAGTTGG } \\
\text { ATCCAACTTTAAGATCCTTTGTTC -3, }\end{array}$ & $\begin{array}{l}426- \\
444\end{array}$ \\
\hline RNAi3 & $\begin{array}{l}\text { 5'-AAAAGGCCAAGCAGGATGCAGCTTGG } \\
\text { ATCCAAGCTGCATCCTGCTTGGCC -3' }\end{array}$ & $\begin{array}{l}1677- \\
1696\end{array}$ \\
\hline
\end{tabular}

TABLE II: PRIMER SEQUENCES FOR RT-QPCR

\begin{tabular}{|l|l|}
\hline Gene & Sequence \\
\hline ADAR1 & Forward primer: \\
& 5'-AGCTCCGCACCAAGGTGGAGAAC-3' \\
& Reverse primer: \\
& 5'-CAGGACATGGTACGGAGTCTC-3' \\
& (GenBank NM_001111) \\
\hline$\beta$-Actin & Forward primer: \\
& 5'-ATCGTGCGTGACATTAAGGAGAAG-3' \\
& Reverse primer: \\
& 5'-AGGAAGGAAGGCTGGAAGAGTG-3' \\
& (GenBank NM_001101.3) \\
\hline POMC & Forward primer: \\
& 5'-CTACGGCGGTTTCATGACCT-3' \\
& Reverse primer: \\
& 5'-CCCTCACTCGCCCTTCTTG-3' \\
& (GenBank NM_001035256) \\
\hline
\end{tabular}

\section{Statistical Analysis}

All of the data in the present study were obtained as averages of experiments that were performed at least in triplicate, and are expressed as means $\pm \mathrm{SD}$; statistical analysis was performed via the Student's $t$-test. A value of $p<$ 0.05 was considered to be statically significant.

\section{RESULTS AND DiSCUSSION}

\section{A. ADARl Knockdown in HaCaT Cells}

In a previous study, we confirmed that $\mathrm{HaCaT}$ cells express ADAR1 via the RT-qPCR method (data not shown). To investigate the effects of ADAR1 mutations on the signal transduction pathway of melanogenesis in advance, we used the RNAi method to knockdown ADAR1 in human keratinocyte $\mathrm{HaCaT}$ cells. Three RNAi target sites were selected, which validated the efficiency of silencing ADAR1 in HaCaT cells. The cells that were separately transfected with RNAi1, RNAi2, or RNAi3 were detected by RT-qPCR 
post-transfection, respectively. The results are shown in Fig. 1a. The silencing efficiency of the three RNAi target sites varied substantially. Both RNAi1 (gene position 2974-2992) and RNAi3 (gene position 1677-1696) resulted in a significant down regulation of ADAR1 gene expression $(\mathrm{P}<$ $0.05)$, where RNAi-3 achieved the highest silencing efficacy of $33.47 \pm 0.2 \%$ of the basal level on mRNA. This phenomenon was believed to be associated with the positional effects, and the result is in agreement with a previous report [13]. In advance, we repeated three batch transfection experiments, termed RNAi3-1, RNAi3-2, and RNAi3-3, using RNAi3 recombinant plasmid, and we determined the silencing efficiency of the transfected cells. The results are shown in Fig. 1b, where all three batch transfection experiments showed significant silencing ADAR1 efficiency $(\mathrm{P}<0.05)$. For comparison purposes, RNAi3-1 and RNAi3-2, which silenced ADAR1 expression to $55.29 \pm 0.4 \%$ and 6.67 $\pm 2.4 \%$ of the basal level, respectively, were selected for further experiments.

(a)

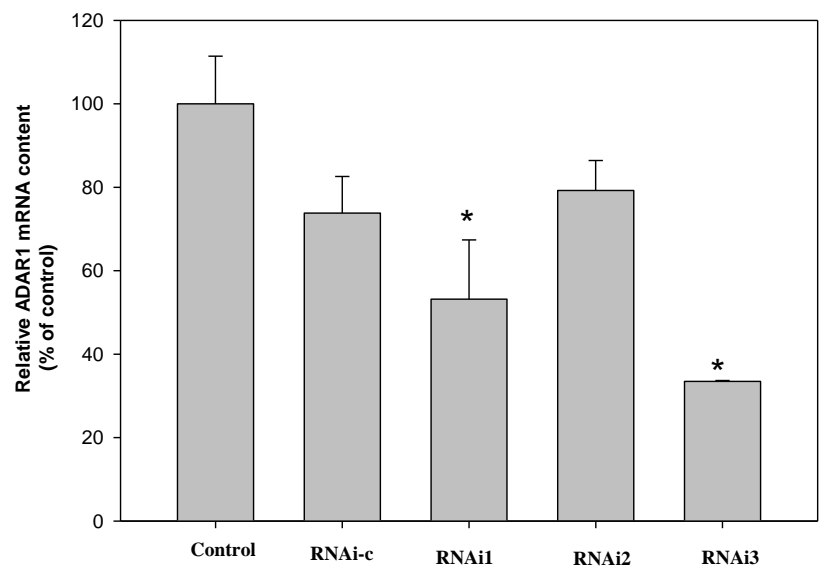

(b)

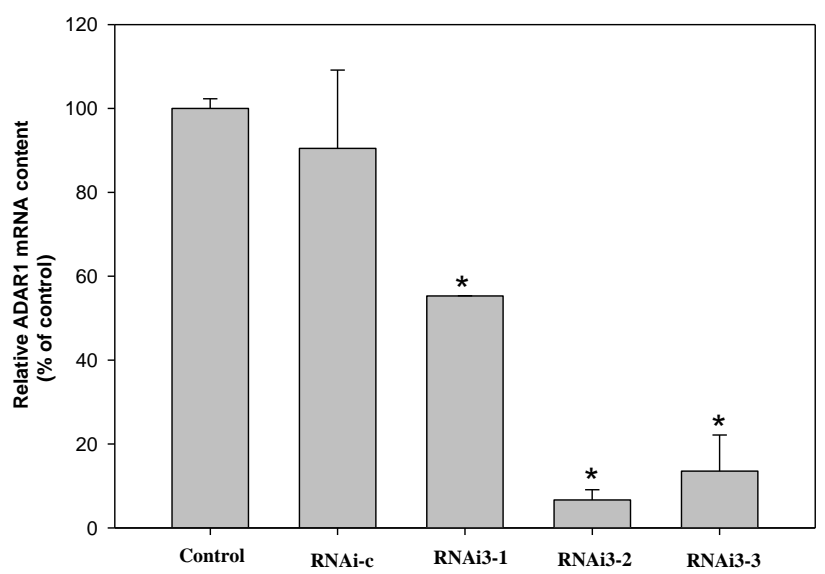

Fig. 1. ADAR1 knockdown in HaCaT cells by RNAi1 to RNAi3 (a) and RNAi3-1 to RNAi3-3 (b). HaCaT cells were transfected with PacI-linearized recombinant pRNAi plasmids, including RNAi-c and RNAi1 to RNAi3 (A) or RNAi-c and RNAi3-1 to RNAi3-3 (B), using PolyJet ${ }^{\mathrm{TM}}$ In Vitro DNA Transfection Reagent, according to the manufacturer's protocol. The transfected cells analyzed the ADAR1 expression via RT-qPCR, as described in Materials and Methods.

\section{B. POMC Expression in ADARl-Knockdown HaCaT Cells}

In order to determine whether ADAR1 is related to melanogenesis, we measured levels of POMC mRNA via RT-qPCR in ADAR1-knockdown HaCaT cells. The results are shown in Fig. 2. For transfected cells by RNAi3-2, which silenced ADAR1 expression to $6.67 \%$ of the basal level, POMC expression in the cells showed a significant reduction in mRNA level $(38.63 \pm 12.6 \%$; $\mathrm{P}<0.05)$, compared to the control. On the other hand, the cells derived from RNAi3-1, which performed a silencing efficacy of only $55.29 \%$, showed only a slight and non-significant reduction $(74.59 \pm 18.7 \%$; $\mathrm{P}$ $=0.23$ ) in POMC expression. In a contrast, the cells derived from negative control RNAi-c, did not show any reduction in POMC expression. The results demonstrate that ADAR1 knockdown reduces POMC expression, in a dose-dependent manner, in $\mathrm{HaCaT}$ cells.

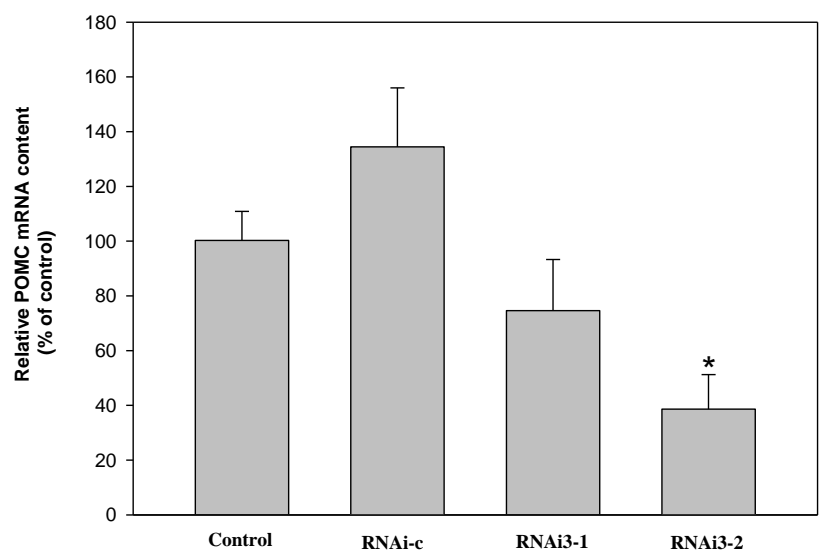

Fig. 2. ADAR1 knockdown inhibits POMC expression in HaCaT cells. The transfected cells by RNAi-c, RNAi3-1, and RNAi3-2 obtained from the experiments of Fig. 1 analyzed the POMC expression via RT-qPCR, as described in Materials and Methods.

Until now, the exact molecular pathogenesis of DSH has not been clarified. Cutaneous biopsies of the hypo- and hyper-pigmented areas taken from DSH patients have shown a reduction of melanin in the basal layer of the hypopigmented zone, and an increase in the melanin pigment of the hyperpigmented zone at the epidermal level, with a normal number of melanocytes [14]. In the histopathological study, hypopigmented areas reveal a decrease or absence of melanin in the basal layer. In contrast, the hyperpigmented areas contain the same number of melanocytes, but with high metabolic activity and a high concentration of melanosomes with normal characteristics. For this reason, it can be assumed that DSH is caused by the dysfunctional regulation of melanogenesis in skin cells, and the disease gene ADAR1 might be involved in regulating melanogenesis. In fact, the ADAR1 gene has been known to play an important role in the regulatory functions of epidermal skin cells [10]. In the present study, our results clearly demonstrate that ADAR1 knockdown inhibits POMC expression in $\mathrm{HaCaT}$ cells. Based on the key role of POMC in the UV-induced pigmentation pathway of human skin keratinocytes, the results open a new avenue to investigate the molecular mechanism of ADAR1 on DSH disease.

\section{REFERENCES}

[1] H. A. Hundley and B. Bass, "ADAR editing in double-stranded UTRs and other noncoding RNA sequences," Trends in Biochem. Sci., vol 35, 2010, pp. 377-383. 
[2] K. Nishikura, "Functions and regulation of RNA editing by ADAR deaminases," Апnи. Rev. Biochem., vol. 79, 2010, pp. 321-349.

[3] B. Zinshteyn and K. Nishikura, "Adenosine-to-inosine RNA editing," Wiley Interdiscip. Rev. Syst. Biol. Med., vol. 1, 2009, pp. 202-209.

[4] S. Maas, Y. Kawahara, K. M. Tamburro, and K. Nishikura, "A-to-I RNA editing and human disease," RNA Biol., vol. 3, 2006, pp. 1-9.

[5] X. J. Zhang, M. Gao, M. Li et al., "Identification of a locus for dyschromatosis symmetrica hereditaria at chromosome 1q11-1q21," $J$. Invest. Dermatol., vol. 120, 2003, pp. 776-780.

[6] Y. Miyamura, T. Suzuki, M. Kono et al., "Mutations of the RNA-specific adenosine deaminase gene (DSRAD) are involved in dyschromatosis symmetrica hereditaria," Am. J. Hum. Genet., vol. 73, 2003, pp. 693-699.

[7] Q. Wang, J. Khillan, P. Gadue, and K. Nishikura, "Requirement of the RNA editing deaminase ADAR1 gene for embryonic erythropoiesis," Science, vol. 290, 2000, pp. 1765-1768.

[8] Q. Wang, M. Miyakoda, W. Yang et al., "Stress-induced apoptosis associated with null mutation of ADAR1 RNA editing deaminase gene," J. Biol. Chem., vol. 279, 2004, pp. 4952-4961.
[9] R. Sharma, Y. Wang, P. Zhou et al., "An essential role of RNA editing enzyme ADAR1 in mouse skin," J. Dermatol. Sci., vol 64, 2001, pp. 70-72.

[10] H. Liu, X. A. Fu, Y. X. Yu et al., "Identification of two novel splice mutations of the ADAR1 gene in two Chinese families with dyschromatosis symmetrica hereditaria," Clini. Exp. Dermatol., vol. 36, 2001, pp. 797-799.

[11] T. S. Chang, "An updated review on tyrosinase inhibitors," Int. J. Mol. Sci., vol 10, 2009, pp. 2440-2475.

[12] G. Imokawa, "Autocrine and paracrine regulation of melanocytes in human skin and in pigmentary disorders," Pig. Cell Res., vol. 17, 2004, pp. 96-110.

[13] T. P. Prakash, C. R. Allerson, P. Dande et al., "Positional effect of chemical modifications on short interference RNA activity in mammalian cells," J. Med. Chem., vol. 48, 2005, pp. 4247-4253.

[14] J. Consigli, M. S. G. Zanni, L. Ragazzini, and C. Danielo, "Dyschromatosis symmetrica hereditaria: report of a sporadic case," Inter. J. Dermatol., vol. 49, 2010, pp. 918-920. 